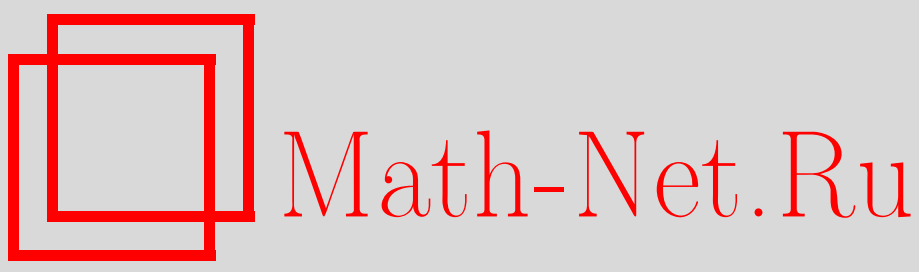

А. М. Степин, А. М. Еременко, Неединственность включения в поток и обширность централизатора для типичного сохраняющего меру преобразования, Матем. сб., 2004, том 195, номер 12, 95-108

DOI: https://doi.org/10.4213/sm867

Использование Общероссийского математического портала Math-Net.Ru подразумевает, что вы прочитали и согласны с пользовательским соглашением

http://www.mathnet.ru/rus/agreement

Параметры загрузки:

IP: 44.207 .124 .84

26 апреля 2023 г., 14:36:00 
УДК $517.987 .5+938.5$

\author{
А. М. Степин, А.М. Еременко
}

\title{
Неединственность включения в поток и обширность централизатора для типичного сохраняющего меру преобразования
}

\begin{abstract}
Для сохраняющего меру преобразования рассматривается задача о включении в потоки. Оказьвается, что для преобразования $T$ с однократньм спектром множество потоков, включающих $T$, если и не пусто, то состоит либо из единственного элемента, либо из бесконечного числа спектрально неэквивалентных потоков.

Доказано, что в типичном случае имеет место максимальная неединственность включения в поток в том смысле, что централизатор типичного преобразования содержит подгруппу, изоморфную бесконечномерному тору. Доказательство этого утверждения использует так назьваемую динамическую альтернативу, топологический аналог теоремы Фубини, фундаментальный факт дескриптивной теории множеств о почти открытости аналитических множеств и лемму Догерти, дающую достаточные условия того, что образ сепарабельного метрического пространства имеет вторую категорию.
\end{abstract}

Библиография: 17 названий.

\section{$\S 1$. Введение}

\section{1. Предварительные сведения.}

Пусть $(X, \mu)$ - вероятностное пространство, обладающее счетньм базисом и полное $\bmod 0$ относительно него. Обозначим через $\operatorname{Aut}(X, \mu)$ группу преобразований пространства $X$, сохраняющих меру $\mu$. Везде далее мы предполагаем, что $(X, \mu)$ изоморфин промежутку $[0,1)$ со стандартной мерой Лебега.

Корнем степени $n$ из $T \in \operatorname{Aut}(X, \mu)$ называется такое сохраняющее меру преобразование $S$, что $S^{n}=T$. Необходимое и достаточное условие существования корня из эргодического преобразования с точечным спектром было найдено П.Р. Халмошем в работе 1942 года. Эта публикация (см. также [1]) поставила вопрос о существовании преобразований с непрерывным спектром, не имеющих корней. Конструкция таких преобразований была анонсирована одним из авторов настоящей статьи в [2] и на Международном конгрессе математиков (Москва, 1966 г.). Примеры, доставляемые конструкцией из [2] и ее обобшениями, являются групповыми расширениями и обладают несчетным централизатором. Ответ на вопрос Халмоша был получен также Р. Чеконом [3], пример перемешиваюшего преобразования без корней предложил Д. Орнстейн [4].

В [2] показано, что число спектрально неэквивалентных корней простой степени $p$ из преобразования $T$ с однократным спектром равно $p^{n}$ для некоторого

Работа выполнена в рамках Программы поддержки ведущих научных школ РФ (грант № HШ-457.2003.1). 
$n=0,1,2, \ldots$, если, разумеется, конечно множество корней из $T$ степени $p$. Имеется обобщение этого утверждения на случай корней произвольной степени. Как само утверждение, так и упомянутое его обобщение перестают быть верньми для преобразований со спектром кратности больше 1.

В контексте задачи о включении преобразований в потоки аналогичное утверждение может быть сформулировано (ср. [5]) следующим образом; здесь и ниже включение $T$ в поток $\left\{F_{t}, t \in \mathbb{R}\right\}$ означает, что $F_{1}=T$.

Теорема 1.1. Если преобразование $T$ с однократным спектром допускает включение в поток, то либо такое включение единственно, либо множество $\mathscr{F}(T)$ потоков, включающих $T$, бесконечно, причем геометрически различные элементы $\mathscr{F}(T)$ спектрально неэквивалентны.

ДокАЗАтЕльство. Действительно, допустим, что $T$ обладает однократным спектром и включается в потоки $\left\{F_{t}\right\}$ и $\left\{G_{t}\right\}$. Тогда эти потоки перестановочны между собой и $\left\{S_{t}=F_{t} G_{-t}, t \in \mathbb{R}\right\}$ есть коммутируюшее с $T$ действие окружности. Но в этом случае

$$
\forall n \in \mathbb{N} \quad\left\{F_{t} S_{n t}\right\} \in \mathscr{F}(T),
$$

а значит, множество $\mathscr{F}(T)$ по крайней мере счетно. Предположим теперь, что спектрально эквивалентные потоки $\left\{F_{t}^{1}\right\}$ и $\left\{F_{t}^{2}\right\}$ включают $T$, и обозначим через $\Phi_{t}^{i}$ унитарные операторы $f(x) \mapsto f\left(F_{t}^{i} x\right), f(x) \in L^{2}(X, \mu), i=1,2$. Для всех $t \in \mathbb{R}$ имеем равенство $\Phi_{t}^{1}=V \Phi_{t}^{2} V^{-1}$, где $V$ - некоторьй унитарный оператор. Отсюда следует, что $V$ коммутирует с оператором $U_{T}: f(x) \mapsto f(T x)$. Поскольку централизатор оператора с однократным спектром абелев, $V$ коммутирует с операторами $\Phi_{t}^{i}, t \in \mathbb{R}, i=1,2$. Таким образом, $\Phi_{t}^{1}=\Phi_{t}^{2}$ для всех $t \in \mathbb{R}$.

Новые результаты в задачах об извлечении корней из сохраняющих меру преобразований, включении таких преобразований в потоки, типичном числе (мощности множеств) корней и включений в потоки связаны с использованием некоторых свойств аналитических ${ }^{1}$ и массивных (всюду плотных типа $G_{\delta}$ ) подмножеств в топологических пространствах. Среди них - фундаментальный факт дескриптивной теории множеств, доказательство которого основано на конструкции "решета Лузина".

ТЕОРема 1.2 (см., например, [6]). Каждое аналитическое подмножество полного сепарабельного метрического пространства обладает свойством Бәра, т.е. представимо в виде симметрической разности $O \triangle M$, әде Ооткрытое множество, а $M$ - первой категории.

Этот факт в сочетании с “динамической альтернативой” был использован Джонатаном Кингом в доказательстве типичности свойства преобразований обладать корнем любой степени [7].

ПрЕДЛОЖЕНИЕ 1.1 (динамическая альтернатива, см. [8]). Пусть Г - груnпа гомеоморфизмов полного сепарабельного метрического пространства $\mathscr{X}$, имеющая плотную орбиту. Тогда всякое множество $M \subset \mathscr{X}$, обладающее свойством Бәра и инвариантное относительно Г, либо массивно, либо имеет первую категорию.

\footnotetext{
${ }^{1}$ Аналитическое множество - это образ борелевского множества при непрерьвном отображении между полньми сепарабельньми пространствами.
} 
Доказательство предложения основано на единственности регулярного представления множества, обладающего свойством Бэра (т.е. такого представления в виде $O \triangle M$, где $M$ - первой категории, а $O$ совпадает с множеством внутренних точек своего замькания, см. [9]).

1.2. Основная теорема. B $\operatorname{Aut}(X, \mu)$ или, более общо, в множестве представлений некоторой локально компактной группы преобразованиями из $\operatorname{Aut}(X, \mu)$ стандартньм образом вводится слабая топология. Типичность действий группы на пространстве $(X, \mu)$, обладающих тем или иным свойством (т.е. массивность множества таких действий), понимается в смысле этой топологии. Существенно, что слабая топология метризуема и соответствующее метрическое пространство полное.

Сформулируем теперь наш основной результат.

Теорема 1.3. Для типичного преобразования $T \in \operatorname{Aut}(X, \mu)$ множество $\mathscr{F}(T)$ потоков, включающих $T$, имеет мощность континуума, причем потоки из $\mathscr{F}(T)$ спектрально неэквивалентны.

Этот результат получен с помощью следующего факта, который является топологическим аналогом теоремы Фубини из теории меры.

ТЕОРема 1.4 (см. [6] или [9]). Пусть $\mathscr{X}, \mathscr{Y ~ - ~ п о л н ы е ~ с е п а р а б е л ь н ы е ~ м е т р и - ~}$ ческие пространства. Если $E$-массивное подмножество в $\mathscr{X} \times \mathscr{Y ~}$, то для типичного $x \in \mathscr{X}$ мнохсество $E_{x}=\{y \in \mathscr{Y}:(x, y) \in E\}$ массивно в $\mathscr{Y}$.

Пусть $T \in \operatorname{Aut}(X, \mu)$ и функция $f: X \rightarrow \mathbb{Z}_{2}=\{0,1\}$ измерима. Косым произведением (надстройкой) с базой $T$ и коииклом $f$ назьвается преобразование

$$
T^{f}(x, n)=(T x, f(x)+n)
$$

пространства $(Y, \nu)=(X, \mu) \times\left(\mathbb{Z}_{2}, \mu_{2}\right)$, где $\mu_{2}(0)=\mu_{2}(1)=\frac{1}{2}$. Косое произведение с базой $T$ мы будем также обозначать через $\widetilde{T}$, не указывая коцикл явно.

Преобразование $R \in \operatorname{Aut}(Y, \nu)$ является косьм произведением в том и только том случае, когда $R$ коммутирует со сдвигом на образующий $(x, j) \mapsto(x, j+1)$. Группу всех косых произведений обозначим $\operatorname{SAut}(Y, \nu)$. Она замкнута в $\operatorname{Aut}(Y, \nu)$ и, стало быть, является полным метрическим пространством, изометричным произведению $\operatorname{Aut}(X, \mu)$ и пространства коциклов $\mathbb{Z}_{2}(X)$ (топология в $\mathbb{Z}_{2}(X)$ вводится с помощью метрики $\left.d_{c}(f, g)=\mu\{x \in X: f(x) \neq g(x)\}\right)$. Именно $\operatorname{Aut}(X, \mu)$ и $\mathbb{Z}_{2}(X)$ будут служить в качестве сомножителей $\mathscr{X}$ и $\mathscr{Y}$ соответственно в теореме 1.4 , а роль $E$ будет играть массивное множество надстроек, допускающих включение в поток.

ПРЕДЛОЖЕНИЕ 1.2. Множество

$$
\mathscr{K}=\left\{T^{f} \in \operatorname{SAut}(Y, \nu): \mathscr{F}\left(T^{f}\right) \neq \varnothing\right\}
$$

массивно в $\operatorname{SAut}(Y, \nu)$.

Следующее предложение содержит альтернативу относительно включения в поток для типичного преобразования. 
ПРЕДЛОЖЕНИЕ 1.3. Пусть преобразование $T$ принадлежит множеству $\mathrm{CAAut}(X, \mu)$ преобразований, допускающих ииклическую аппроксимацию ${ }^{2}$ со скоростью о $(1 / n)$, и $\mathscr{F}(T)$ не более чем счетно. Тогда мнохсество $\mathscr{K}_{T}$ коииклов $f$, для которых $\mathscr{F}\left(T^{f}\right) \neq \varnothing$, имеет первую категорию в $\mathbb{Z}_{2}(X)$.

Пусть предложения 1.2 и 1.3 доказаны. Для типичного преобразования $T$ множество $\mathscr{K}_{T}$ массивно в пространстве коциклов по предложению 1.2 и теореме 1.4. Множество CAAut $(X, \mu)$ массивно в $\operatorname{Aut}(X, \mu)$, поэтому в силу предложения 1.3 для типичного $T \in \operatorname{Aut}(X, \mu)$ множество $\mathscr{F}(T)$ несчетно. Поскольку $\mathscr{F}(T)$ - замкнутое множество (как прообраз $T$ при непрерывном отображении $\left\{T_{t}\right\} \mapsto T_{1}$ ), оно имеет мощность континуума (см., например, [6]), что доказывает теорему 1.3.

\section{§ 2. Типичность надстроек, включаемых в потоки}

2.1. Редукция к динамической альтернативе. Пусть $\xi^{n}$ обозначает разбиение промежутка $X$ на $2^{n}$ равных полуинтервалов, $\widetilde{\xi}^{n}=\xi^{n} \times \varepsilon_{2}$, где $\varepsilon_{2}-$ разбиение $\mathbb{Z}_{2}$ на точки. Слабую топологию в пространстве $\operatorname{Aut}(Y, \nu)$ зададим с помощью метрики

$$
d_{Y}(T, S)=\sum_{m=1}^{\infty} \frac{1}{2^{m}} d_{Y}^{m}(T, S)
$$

где

$$
d_{Y}^{m}(T, S)=\frac{1}{2} \sum_{C \in \widetilde{\xi}^{m}}\left(\mu(T C \triangle S C)+\mu\left(T^{-1} C \triangle S^{-1} C\right)\right)
$$

Функция

$$
d_{F}\left(\left\{F_{t}\right\},\left\{G_{t}\right\}\right)=\sup _{s \in[0,1]} d_{Y}\left(F_{s}, G_{s}\right)
$$

превращает множество Flow $(Y, \nu)$ всех потоков на $(Y, \nu)$ в полное метрическое пространство.

УТВЕРЖДЕНИЕ 2.1. Множество

$$
\operatorname{SFlow}(\mathrm{Y}, \nu)=\left\{\left\{F_{t}\right\} \in \operatorname{Flow}(Y, \nu): \forall t \in \mathbb{R} \quad F_{t} \in \operatorname{SAut}(Y, \nu)\right\}
$$

является полным сепарабельным пространством относительно топологии, индуиированной метрикой $d_{F}$.

Рассмотрим непрерьвное отображение $\mathbb{P}: \operatorname{SFlow}(\mathrm{Y}, \nu) \rightarrow \operatorname{SAut}(Y, \nu)$, сопоставляющее потоку $\left\{F_{t}\right\}$ преобразование $F_{1}$. Множество $\mathscr{K}$ (см. предложение 1.2$)$ является образом пространства $\operatorname{SFlow}(\mathrm{Y}, \nu)$ при этом отображении, в силу утверждения 2.1 и теоремы 1.2 оно обладает свойством Бэра. Воспользуемся теперь предложением 1.1, для чего рассмотрим действие группы $\operatorname{SAut}(Y, \nu)$ на себе сопряжениями. Множество $\mathscr{K}$ инвариантно относительно этого действия.

Лемма 2.1 (о сопряженности). Пусть $T \in \operatorname{Aut}(X, \mu)$ - апериодическое преобразование, $f \in \mathbb{Z}_{2}(X)$. Тогда множество $\left\{\widetilde{Q}^{-1} T^{f} \widetilde{Q}: \widetilde{Q} \in \operatorname{SAut}(Y, \nu)\right\}$ плотно в $\operatorname{SAut}(Y, \nu)$.

\footnotetext{
${ }^{2}$ По поводу соответствующих определений см. [10].
} 
Доказательство приведено в $\S 3$. Согласно этой лемме указанное вьше действие имеет плотную орбиту, следовательно, множество $\mathscr{K}$ либо массивно, либо первой категории. Для того чтобы исключить вторую возможность, нам потребуется следующее понятие.

Точка $x \in \mathscr{X}$ называется точкой локальной плотности непрерывного отображения $f: \mathscr{X} \mapsto \mathscr{Y}$, если для любой окрестности $U \ni x$ найдется окрестность $V \ni f(x)$ такая, что $f(U)$ плотно в $V$. Множество точек локальной плотности данного отображения обозначается $\operatorname{Loc} \operatorname{Den}(f)$.

Лемма 2.2 (Догерти, см. [7]). Пусть $f: \mathscr{X} \mapsto \mathscr{Y ~ - ~ н е п р е р ы в н о е е ~ о т о б р а - ~}$ жение и $\mathscr{X}$ является полным сепарабельным метрическим пространством. Eсли $\operatorname{LocDen}(f)$ плотно в $\mathscr{X}$, то $f(\mathscr{X})$ не может иметь первую категорию $\boldsymbol{\beta} \mathscr{Y}$.

Таким образом, предложение 1.2 будет доказано, когда мы установим, что множество LocDen $(\mathbb{P})$ всюду плотно в $\operatorname{SFlow}(\mathrm{Y}, \nu)$. Наше доказательство будет следовать схеме рассуждений из [11].

Зафиксируем $\alpha \in(0,1)$ и рассмотрим косое произведение $P_{\alpha}^{f}$ с базой $P_{\alpha}: x \mapsto$ $x+\alpha(\bmod 1)$ и коциклом

$$
f(x)= \begin{cases}0, & x \in[0,1-\alpha) \\ 1, & x \in[1-\alpha, 1)\end{cases}
$$

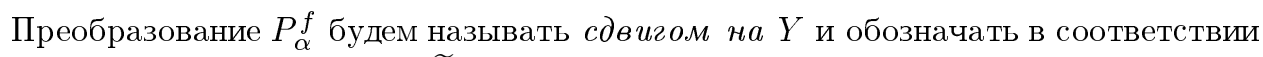
с договоренностью просто $\widetilde{P}_{\alpha}$. При изоморфизме пространств $(X, \mu)$ и $(Y, \nu)$ посредством отображения $(x, j) \mapsto y=(x+j) / 2$ автоморфизму $\widetilde{P}_{\alpha}$ соответствует преобразование сдвига $y \mapsto y+\alpha / 2$.

Рассмотрим множество $\mathscr{P}$ потоков $\left\{\widetilde{F}_{t}\right\} \in \operatorname{SFlow}(\mathrm{Y}, \nu)$, для которых преобразование $\widetilde{F}_{1}$ сопряжено иррациональному сдвигу на $Y$ с помощью косого произведения, т.е.

$$
\widetilde{F}_{1}=\varphi \widetilde{P}_{\alpha} \varphi^{-1}, \quad \alpha \in \mathbb{R} \backslash \mathbb{Q}, \quad \varphi \in \operatorname{SAut}(Y, \nu) .
$$

УТВЕРЖДЕНИЕ 2.2. Справедливо включение $\mathscr{P} \subset \operatorname{LocDen}(\mathbb{P})$.

УТВеРЖДЕнИЕ 2.3. Множество $\mathscr{P}$ плотно в $\mathrm{SFlow}(\mathrm{Y}, \nu)$.

\section{2. Леммы для вывода утверждения 2.2 .}

Лемма 2.3. Пусть $\left\{\widetilde{F}_{t}\right\} \in \mathscr{P}$. Тогда для любого $n \in \mathbb{N}\left\{\widetilde{F}_{2^{-n} t}\right\} \in \mathscr{P}$.

ДокАЗАТЕЛЬСтво. Достаточно проверить, что если $\left\{\widetilde{F}_{t}\right\} \in \mathscr{P}$, то преобразование $\widetilde{F}_{1 / 2}$ сопряжено иррациональному сдвигу посредством некоторого $\varphi_{1} \in$ $\operatorname{SAut}(Y, \nu)$. Преобразование $\widetilde{F}_{1 / 2}$, как и $\widetilde{F}_{1}$, обладает чисто точечным спектром и, стало быть, имеет вид $\varphi_{1} \widetilde{P}_{\beta} \varphi^{-1}$. Отсюда следует, что $\varphi^{-1} \varphi_{1}$ коммутирует с $\widetilde{P}_{\alpha}$. Сдвиг $\widetilde{P}_{\alpha}$ имеет однократный спектр, поэтому $\varphi^{-1} \varphi_{1}$ является косым произведением вида (1), следовательно, $\varphi_{1} \in \operatorname{SAut}(Y, \nu)$, что доказывает лемму.

Занумеруем элементы разбиения $\xi^{n} \times\{0\}$ слева направо числами от 0 до $2^{n}-1$ и аналогично элементы $\xi^{n} \times\{1\}$ - числами от $2^{n}$ до $2^{n+1}-1$. 
ОПРЕДЕЛЕНИЕ 2.1. Назовем $(2, n)$-ииклом преобразование, реализующее на элементах $\widetilde{\xi}^{n}$ посредством параллельного переноса перестановку вида $\sigma_{1} \sigma_{2}$, где $\sigma_{1}, \sigma_{2}$ - непересекающиеся циклы длины $2^{n}$, сопряженные с помощью "сдвига на образующий” $\theta=\left(02^{n}\right)\left(12^{n}+1\right) \cdots\left(2^{n}-12^{n+1}-1\right)$ :

$$
\theta \sigma_{1}=\sigma_{2} \theta
$$

Отметим, что всякий $(2, n)$-цикл $S$ заведомо является косьм произведением вида (1), так как

$$
\theta S=\theta \sigma_{1} \sigma_{2}=\sigma_{2} \theta \sigma_{2}=\sigma_{2} \sigma_{1} \theta=S \theta
$$

$(2, n)$-циклы составляют в $\operatorname{SAut}(Y, \nu)$ всюду плотное подмножество.

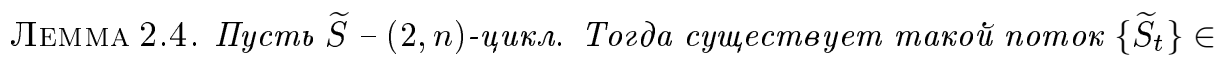
$\operatorname{SFlow}(\mathrm{Y}, \nu)$, чmo $\widetilde{S}_{1}=\widetilde{S} u$

$$
\forall t \in[0,1] \quad d_{Y}\left(\widetilde{S}_{t}, \mathrm{id}\right) \leqslant d_{Y}(\widetilde{S}, \mathrm{id})
$$

id - тождественное преобразование.

ДоказАтЕльство. Найдется такое $\varphi \in \operatorname{SAut}(Y, \nu)$, что $\widetilde{S}=\varphi \widetilde{Q} \varphi^{-1}$, где $\widetilde{Q}-$ $(2, n)$-цикл вида

$$
\left(01 \ldots 2^{n}-1\right)\left(2^{n} 2^{n}+1 \ldots 2^{n+1}-1\right)
$$

Действительно, если перестановка, соответствуюшая $\widetilde{S}$, есть

$$
\begin{gathered}
\left(k_{0} k_{1} \ldots k_{2^{n}-1}\right)\left(k_{2^{n}} k_{2^{n}+1} \ldots k_{2^{n+1}-1}\right), \\
k_{0}=0, \quad k_{i+2^{n}}=k_{i}+2^{n}\left(\bmod 2^{n+1}\right), \quad i=0, \ldots, 2^{n}-1,
\end{gathered}
$$

то в качестве $\varphi$ нужно взять преобразование, которое параллельным переносом переводит элемент разбиения $\widetilde{\xi}^{n}$ с номером $i$ в элемент с номером $k_{i}, i=0, \ldots$, $2^{n+1}-1$.

Положим $\widetilde{Q}_{t}(x, j)=\left(x+t / 2^{n}(\bmod 1), j\right)$ и $\widetilde{S}_{t}=\varphi \widetilde{Q}_{t} \varphi^{-1}$. Ясно, что $\widetilde{S}_{1}=\widetilde{S}$. Для того чтобы проверить неравенство (3), убедимся, что для всякого элемента $C$ разбиения $\widetilde{\xi}^{m}, m=1,2, \ldots$, выполнено

$$
\forall t \in[0,1] \quad \nu\left(\widetilde{S}_{t} C \cap C\right) \geqslant \nu(\widetilde{S} C \cap C)
$$

Если $m \geqslant n$, то $\widetilde{S} C \cap C=\varnothing$. Пусть теперь $m<n$. Тогда

$$
\nu(\widetilde{S} C \cap C)=\frac{1}{2 \cdot 2^{n}} \operatorname{card}\left\{B \in \widetilde{\xi}^{n}: B \subset C \text { и } \widetilde{S} B \subset C\right\} .
$$

При $t \in[0,1]$ для любого элемента $B \in \widetilde{\xi}^{n}$ вьполняется $\widetilde{S}_{t} B \subset(B \cup \widetilde{S} B)$. Следовательно, если $B \subset C$ и $\widetilde{S} B \subset C$, то $\widetilde{S}_{t} B \subset C$, а значит, (4) имеет место. 
Лемма 2.5. Пусть $\alpha \in \mathbb{R} \backslash \mathbb{Q}, \widetilde{T}=\varphi \widetilde{P}_{\alpha} \varphi^{-1}, \varphi \in \operatorname{SAut}(Y, \nu)$, и дано $\varepsilon>0$. Тогда существуют такие $\delta>0$ и $N \in \mathbb{N}$, ито для всякого $(2, n)$-иикла $\widetilde{S}$, $n>N$, удовлетворяющего условию

$$
d_{Y}\left(\widetilde{T}^{2}, \widetilde{S}\right)<\delta,
$$

найдется $(2, n+1)-$ цикл $\widetilde{S}_{1}$ такой, что $\widetilde{S}_{1}^{2}=\widetilde{S}$ u

$$
d_{Y}\left(\widetilde{T}, \widetilde{S}_{1}\right)<\varepsilon .
$$

ДокАЗАТЕЛЬСтво. Преобразование $\widetilde{T}^{2}$ сопряжено иррациональному сдвигу $\widetilde{P}_{2 \alpha}$, поэтому найдется $k$ такое, что

$$
d_{Y}\left(\widetilde{T},\left(\widetilde{T}^{2}\right)^{k}\right)<\varepsilon .
$$

Выберем $N$ из условия

$$
\sum_{n \geqslant N} \frac{1}{2^{n}}<\varepsilon
$$

а $\delta>0$ возьмем настолько малым, что для любого преобразования $\widetilde{S} \in \operatorname{SAut}(Y, \nu)$, лежашего в $\delta$-окрестности $T^{2}$, выполняется неравенство

$$
d_{Y}\left(\widetilde{S}^{q}, \widetilde{T}^{2 q}\right)<\varepsilon, \quad q=-k+1, k .
$$

Зафиксируем теперь $n>N$ и $(2, n)$-цикл $\widetilde{S}$ из $\delta$-окрестности преобразования $\widetilde{T}^{2}$, переставляющий полуинтервалы разбиения $\widetilde{\xi}^{n}$. Введем следуюшее обозначение: если $C$ - произвольный элемент $\widetilde{\xi}^{n}$, то входящие в него полуинтервалы разбиения $\widetilde{\xi}^{n+1}$ (т.е. левую и правую половины $C$ ) будем обозначать $C_{L}$ и $C_{R}$ соответственно. Положим

$$
\begin{aligned}
\widetilde{S}_{1} C_{R} & =\widetilde{S}^{k} C_{L}, \\
\widetilde{S}_{1} C_{L} & =\widetilde{S}^{-k+1} C_{R}
\end{aligned}
$$

и вычислим $d_{Y}^{m}\left(\widetilde{S}_{1}, \widetilde{T}\right)$ для $m \leqslant n$.

Пусть $A$ - произвольный элемент $\widetilde{\xi}^{m}$, обозначим

$$
A_{R}=A \cap \bigcup_{C \in \widetilde{\xi}^{n}} C_{R}, \quad A_{L}=A \cap \bigcup_{C \in \widetilde{\xi}^{n}} C_{L} .
$$

Тогда

$$
\begin{aligned}
& \widetilde{S}_{1} A_{R} \subset \widetilde{S}^{k} A, \\
& \widetilde{S}_{1} A_{L} \subset \widetilde{S}^{-k+1} A
\end{aligned}
$$

и аналогично

$$
\begin{aligned}
& \widetilde{S}_{1}^{-1} A_{R} \subset \widetilde{S}^{k-1} A \\
& \widetilde{S}_{1}^{-1} A_{L} \subset \widetilde{S}^{-k} A .
\end{aligned}
$$

Следовательно,

$$
\nu\left(\widetilde{S}_{1} A \backslash \widetilde{T} A\right) \leqslant \nu\left(\widetilde{S}^{k} A \backslash \widetilde{T} A\right)+\nu\left(\widetilde{S}^{-k+1} A \backslash \widetilde{T} A\right)
$$

и

$$
\nu\left(\widetilde{S}_{1}^{-1} A \backslash \widetilde{T}^{-1} A\right) \leqslant \nu\left(\widetilde{S}^{-k} A \backslash \widetilde{T}^{-1} A\right)+\nu\left(\widetilde{S}^{k-1} A \backslash \widetilde{T}^{-1} A\right) .
$$


Поскольку $\nu(A \triangle B)=2 \nu(A \backslash B)$, если $\nu A=\nu B$, имеем

$$
\nu\left(\widetilde{S}_{1} A \triangle \widetilde{T} A\right) \leqslant \nu\left(\widetilde{S}^{k} A \triangle \widetilde{T} A\right)+\nu\left(\widetilde{S}^{-k+1} A \triangle \widetilde{T} A\right)
$$

и

$$
\nu\left(\widetilde{S}_{1}^{-1} A \triangle \widetilde{T}^{-1} A\right) \leqslant \nu\left(\widetilde{S}^{-k} A \triangle \widetilde{T}^{-1} A\right)+\nu\left(\widetilde{S}^{k-1} A \triangle \widetilde{T}^{-1} A\right) .
$$

Складывая два последних неравенства и суммируя по $A \in \widetilde{\xi}^{m}$, получаем для $m \leqslant n$

$$
d_{Y}^{m}\left(\widetilde{S}_{1}, \widetilde{T}\right) \leqslant d_{Y}^{m}\left(\widetilde{S}^{k}, \widetilde{T}\right)+d_{Y}^{m}\left(\widetilde{S}^{-k+1}, \widetilde{T}\right) .
$$

Отсюда с учетом (6) получаем неравенство

$$
d_{Y}\left(\widetilde{S}_{1}, \widetilde{T}\right) \leqslant d_{Y}\left(\widetilde{S}^{k}, \widetilde{T}\right)+d_{Y}\left(\widetilde{S}^{-k+1}, \widetilde{T}\right)+\varepsilon .
$$

Однако в силу (5) и (7) выполняется

$$
d_{Y}\left(\widetilde{S}^{k}, \widetilde{T}\right) \leqslant d_{Y}\left(\widetilde{S}^{k}, \widetilde{T}^{2 k}\right)+d_{Y}\left(\widetilde{T}^{2 k}, \widetilde{T}\right)<2 \varepsilon
$$

и аналогично

$$
d_{Y}\left(\widetilde{S}^{-k+1}, \widetilde{T}\right) \leqslant d_{Y}\left(\widetilde{S}^{-k+1}, \widetilde{T}^{2(-k+1)}\right)+d_{Y}\left(\widetilde{T}^{2(-k+1)}, \widetilde{T}\right)<2 \varepsilon,
$$

так как преобразование $\widetilde{T}$ сохраняет меру. Таким образом,

$$
d_{Y}\left(\widetilde{S}_{1}, \widetilde{T}\right)<5 \varepsilon .
$$

2.3. Доказательство утверждения 2.2. Пусть даны $\varepsilon>0$ и $\left\{\widetilde{T}_{t}\right\} \in \mathscr{P}$. Необходимо указать такое $\delta>0$, что для всякого преобразования $\widetilde{S}$ из некоторого плотного подмножества в $\operatorname{SAut}(Y, \nu), d_{Y}\left(\widetilde{S}, \widetilde{T}_{1}\right)<\delta$, найдется поток $\left\{\widetilde{S}_{t}\right\} \in$ $\operatorname{SFlow}(\mathrm{Y}, \nu)$ такой, что $\widetilde{S}_{1}=\widetilde{S}_{\text {и }}$

$$
d_{F}\left(\left\{\widetilde{S}_{t}\right\},\left\{\widetilde{T}_{t}\right\}\right)<\varepsilon .
$$

Выберем $k \in \mathbb{N}$ так, чтобы

$$
\forall r \in\left[0, \frac{1}{2^{k}}\right] \quad d_{Y}\left(\widetilde{T}_{r}, \text { id }\right)<\varepsilon .
$$

Пусть $\omega \in(0, \varepsilon)$ настолько мало, что для любого $\widetilde{S} \in \operatorname{SAut}(Y, \nu)$, лежашего в $\omega$-окрестности преобразования $\widetilde{T}_{1 / 2^{k}}$, выполняется

$$
d_{Y}\left(\widetilde{S}^{m}, \widetilde{T}_{m / 2^{k}}\right)<\varepsilon, \quad m=1,2, \ldots, 2^{k} .
$$

В силу леммы 2.3 преобразование $\widetilde{T}_{1 / 2^{k}}$ сопряжено иррациональному сдвигу на $Y$. Согласно лемме 2.5 существуют $N_{1} \in \mathbb{N}$ и $\delta_{1}>0$ такие, что для всякого $(2, n-1)$ цикла $\widetilde{R}_{1}, n>N_{1}, d_{Y}\left(\widetilde{R}_{1}, \widetilde{T}_{2 / 2^{k}}\right)<\delta_{1}$, найдется $(2, n)$-цикл $\widetilde{R}_{0}$, удовлетворяюший условиям

$$
\widetilde{R}_{0}^{2}=\widetilde{R}_{1}, \quad d_{Y}\left(\widetilde{R}_{0}, \widetilde{T}_{1 / 2^{k}}\right)<\omega .
$$


Аналогично, преобразование $T_{2 / 2^{k}}$ сопряжено иррациональному сдвигу на $Y$, поэтому сушествуют $N_{2}>N_{1}$ и $\delta_{2}>0$ такие, что для всякого $(2, n-2)$-цикла $\widetilde{R}_{2}$, $n>N_{2}, d_{Y}\left(\widetilde{R}_{2}, \widetilde{T}_{2^{2} / 2^{k}}\right)<\delta_{2}$, найдется $(2, n-1)$-цикл $\widetilde{R}_{1}$, удовлетворяющий условиям

$$
\widetilde{R}_{1}^{2}=\widetilde{R}_{2}, \quad d_{Y}\left(\widetilde{R}_{1}, \widetilde{T}_{2 / 2^{k}}\right)<\delta_{1} .
$$

Применив $k$ раз леммы 2.3 и 2.5 , укажем числа $N=N_{k}$ и $\delta=\delta_{k}$ такие, что для всякого $(2, n-k)$-цикла $\widetilde{S}, n>N, d_{Y}\left(\widetilde{S}, \widetilde{T}_{1}\right)<\delta$, найдется $(2, n)$-цикл $\widetilde{R}$ такой, что

$$
\widetilde{R}^{2^{k}}=\widetilde{S}, \quad d_{Y}\left(\widetilde{R}, \widetilde{T}_{1 / 2^{k}}\right)<\omega .
$$

В силу выбора $\omega$ справедливо

$$
d_{Y}\left(\widetilde{R}^{m}, \widetilde{T}_{m / 2^{k}}\right)<\varepsilon, \quad m=1, \ldots, 2^{k} .
$$

Пусть $\widetilde{R}_{t}$ - поток, построенный из $\widetilde{R}$ по лемме 2.4. Положим $\widetilde{S}_{t}=\widetilde{R}_{2^{k} t}$, тогда $\widetilde{S}_{1 / 2^{k}}=\widetilde{R}$ и для $r \in\left[0,1 / 2^{k}\right]$ в силу выбора $\widetilde{R}$ и неравенства (8) вьполняется

$$
d_{Y}\left(\widetilde{S}_{r}, \mathrm{id}\right) \leqslant d_{Y}\left(\widetilde{S}_{1 / 2^{k}}, \mathrm{id}\right) \leqslant d_{Y}\left(\widetilde{R}, \widetilde{T}_{1 / 2^{k}}\right)+d_{Y}\left(\widetilde{T}_{1 / 2^{k}}, \mathrm{id}\right)<2 \varepsilon
$$

Пусть $t$ - произвольное число из отрезка $[0,1], t=m / 2^{k}+r$, где $m$ целое, $0 \leqslant r<$ $1 / 2^{k}$. Благодаря (8)-(10) имеем

$$
\begin{aligned}
d_{Y}\left(\widetilde{S}_{t}, \widetilde{T}_{t}\right) & \leqslant d_{Y}\left(\widetilde{S}_{t}, \widetilde{S}_{m / 2^{k}}\right)+d_{Y}\left(\widetilde{S}_{m / 2^{k}}, \widetilde{T}_{m / 2^{k}}\right)+d_{Y}\left(\widetilde{T}_{m / 2^{k}}, \widetilde{T}_{t}\right) \\
& =d_{Y}\left(\widetilde{S}_{r}, \mathrm{id}\right)+d_{Y}\left(\widetilde{S}_{m / 2^{k}}, \widetilde{T}_{m / 2^{k}}\right)+d_{Y}\left(\mathrm{id}, \widetilde{T}_{r}\right)<4 \varepsilon,
\end{aligned}
$$

поскольку участвуюшие преобразования сохраняют меру.

2.4. Доказательство утверждения 2.3. Пусть даны $\left\{\widetilde{T}_{t}\right\} \in \operatorname{SFlow}(\mathrm{Y}, \nu)$ и $\varepsilon>0$. Выберем такое $k$, чтобы

$$
\forall r \in\left[0, \frac{1}{2^{k}}\right] \quad d_{Y}\left(\widetilde{T}_{r}, \mathrm{id}\right)<\varepsilon,
$$

и $\delta \in(0, \varepsilon)$ настолько малое, чтобы для всякого $\widetilde{S} \in \operatorname{SAut}(Y, \nu)$ из $\delta$-окрестности преобразования $\widetilde{T}_{1 / 2^{k}}$ выполнялось

$$
d_{Y}\left(\widetilde{S}^{m}, \widetilde{T}_{m / 2^{k}}\right)<\varepsilon, \quad m=1,2, \ldots, 2^{k} .
$$

Найдем такое преобразование $S \in \operatorname{Aut}(X, \mu)$, циклически переставляющее посредством параллельного переноса элементы разбиения $\xi^{n}$ подходяшего ранга $n$, и такой $\xi^{n}$-измеримый нечетный ${ }^{3}$ коцикл $f(x)$, чтобы косое произведение $\widetilde{S}=S^{f}$ оказалось в $\delta / 2$-окрестности $\widetilde{T}_{1 / 2^{k}}$. Как в доказательстве леммы 2.4 , можно указать $\varphi \in \operatorname{SAut}(Y, \nu)$ такое, что $\widetilde{S}=\varphi \widetilde{P}_{1 / 2^{n}} \varphi^{-1}$. Положим $\left\{\widetilde{Q}_{t}=\varphi \widetilde{P}_{t / 2^{n}} \varphi^{-1}, t \in \mathbb{R}\right\}$, тогда $\widetilde{Q}_{1}=\widetilde{S}$.

\footnotetext{
${ }^{3}$ То есть такой, сумма mod 2 значений которого по элементам $\xi^{n}$ равняется 1.
} 
Аналогично доказательству леммы (2.4) устанавливается, что

$$
\forall t \in[0,1] \quad d_{Y}\left(\widetilde{Q}_{t}, \mathrm{id}\right) \leqslant d_{Y}\left(\widetilde{Q}_{1}, \mathrm{id}\right) .
$$

Пусть $\alpha$ - иррациональное число из интервала $\left(0,1 / 2^{n}\right)$, положим

$$
\left\{\widetilde{Q}_{t}^{\prime}=\varphi \widetilde{P}_{\alpha t} \varphi^{-1}, t \in \mathbb{R}\right\}
$$

Тогда

$$
\forall t \in[0,1] \quad d_{Y}\left(\widetilde{Q}_{t}^{\prime}, \mathrm{id}\right)<d_{Y}\left(\widetilde{Q}_{1}, \mathrm{id}\right)
$$

и можно выбрать $\alpha$ настолько близким к $1 / 2^{n}$, что

$$
d_{Y}\left(\widetilde{Q}_{1}^{\prime}, \widetilde{Q}_{1}\right)<\frac{\delta}{2}
$$

Наконец, положим $\left\{\widetilde{Q}_{t}^{\prime \prime}=\widetilde{Q}_{2^{k} t}^{\prime}, t \in \mathbb{R}\right\}$, тогда $\left\{\widetilde{Q}_{t}^{\prime \prime}\right\} \in \mathscr{P}$. Покажем, что $\left\{\widetilde{Q}_{t}^{\prime \prime}\right\}$ достаточно хорошо приближает $\left\{\widetilde{T}_{t}\right\}$. Действительно, произвольное $t \in[0,1]$ можно представить в виде $t=m / 2^{k}+r, 0 \leqslant r<1 / 2^{k}, m$ целое. Поскольку рассматриваемые преобразования сохраняют меру, имеем

$$
\begin{aligned}
d_{Y}\left(\widetilde{Q}_{t}^{\prime \prime}, \widetilde{T}_{t}\right) & \leqslant d_{Y}\left(\widetilde{Q}_{t}^{\prime \prime}, \widetilde{Q}_{m / 2^{k}}^{\prime \prime}\right)+d_{Y}\left(\widetilde{Q}_{m / 2^{k}}^{\prime \prime}, \widetilde{T}_{m / 2^{k}}\right)+d_{Y}\left(\widetilde{T}_{m / 2^{k}}, \widetilde{T}_{t}\right) \\
& =d_{Y}\left(\widetilde{Q}_{r}^{\prime \prime}, \mathrm{id}\right)+d_{Y}\left(\widetilde{Q}_{m / 2^{k}}^{\prime \prime}, \widetilde{T}_{m / 2^{k}}\right)+d_{Y}\left(\mathrm{id}, \widetilde{T}_{r}\right) .
\end{aligned}
$$

Последнее слагаемое не превосходит $\varepsilon$ в силу (11). Из (12) следует, что

$$
d_{Y}\left(\widetilde{Q}_{m / 2^{k}}^{\prime \prime}, \widetilde{T}_{m / 2^{k}}\right)<\varepsilon, \quad m=1,2, \ldots, 2^{k},
$$

поскольку в силу (14) и выбора $\widetilde{S}=\widetilde{Q}_{1}$ выполняется неравенство

$$
d_{Y}\left(\widetilde{Q}_{1 / 2^{k}}^{\prime \prime}, \widetilde{T}_{1 / 2^{k}}\right) \leqslant d_{Y}\left(\widetilde{Q}_{1 / 2^{k}}^{\prime \prime}, \widetilde{Q}_{1}\right)+d_{Y}\left(\widetilde{Q}_{1}, \widetilde{T}_{1 / 2^{k}}\right)<\delta .
$$

Наконец, для $r \in\left[0,1 / 2^{k}\right]$ благодаря (13) и (11) выполняется

$$
d_{Y}\left(\widetilde{Q}_{r}^{\prime \prime}, \mathrm{id}\right)<d_{Y}\left(\widetilde{Q}_{1}, \mathrm{id}\right) \leqslant d_{Y}\left(\widetilde{Q}_{1}, \widetilde{T}_{1 / 2^{k}}\right)+d_{Y}\left(\widetilde{T}_{1 / 2^{k}}, \mathrm{id}\right)<2 \varepsilon .
$$

Таким образом,

$$
d_{Y}\left(\widetilde{Q}_{t}^{\prime \prime}, \widetilde{T}_{t}\right)<4 \varepsilon
$$

2.5. Полнота и сепарабельность $\operatorname{SFlow}(\mathrm{Y}, \nu)$.

Полнота. Если последовательность потоков $\left(\left\{\widetilde{S}_{t}\right\}\right)_{n=1}^{\infty}$ является фундаментальной в метрике $d_{F}$, то для каждого фиксированного $t_{0}$ последовательность преобразований $\left(\widetilde{S}_{t_{0}}\right)_{n=1}^{\infty}$ фундаментальна в метрике $d_{Y}$. Так как пространство $\operatorname{SAut}(Y, \nu)$ полно, то предел $\widetilde{S}_{t_{0}}^{\prime}$ этой последовательности существует и является косым произведением вида (1). Ясно, что преобразования $\left\{\widetilde{S}_{t}^{\prime}, t \in \mathbb{R}\right\}$ образуют поток, который и будет пределом исходной последовательности при $n \rightarrow \infty$. Таким образом, $\operatorname{SFlow}(\mathrm{Y}, \nu)$ - полное пространство. 
Сепарабельность. Обозначим через $M$ множество потоков, полученных по лемме 2.4 из всевозможных $(2, n)$-циклов. Докажем, что множество

$$
\bigcup_{k}\left\{\left\{\widetilde{S}_{2^{k} t}\right\}:\left\{\widetilde{S}_{t}\right\} \in M\right\}
$$

является всюду плотным в SFlow $(\mathrm{Y}, \nu)$.

Пусть даны $\left\{\widetilde{T}_{t}\right\} \in \operatorname{SFlow}(\mathrm{Y}, \nu)$ и $\varepsilon>0$. Выберем $k$ такое, что

$$
\forall r \in\left[0, \frac{1}{2^{k}}\right] \quad d_{Y}\left(\widetilde{T}_{r}, \text { id }\right)<\varepsilon,
$$

и $\delta \in(0, \varepsilon)$ настолько малое, чтобы для каждого $\widetilde{S}$ из $\delta$-окрестности преобразования $\widetilde{T}_{1 / 2^{k}}$ выполнялось

$$
d_{Y}\left(\widetilde{S}^{m}, \widetilde{T}_{m / 2^{k}}\right)<\varepsilon, \quad m=1,2, \ldots, 2^{k} .
$$

Пусть $\widetilde{S}-(2, n)$-цикл, достаточно близкий к $\widetilde{T}_{1 / 2^{k}}, \quad\left\{\widetilde{S}_{t}^{\prime}\right\}$ - поток из леммы 2.4 , построенный по $\widetilde{S}$. Положим $\left\{\widetilde{S}_{t}^{\prime \prime}=\widetilde{S}_{2^{k} t}^{\prime}, t \in \mathbb{R}\right\}$. Благодаря (15) для каждого $m=1,2, \ldots, 2^{k}$ вьполнено неравенство $d_{Y}\left(\widetilde{S}_{m / 2^{k}}^{\prime \prime}, \widetilde{T}_{m / 2^{k}}\right)<\varepsilon$. В силу выбора $k$ для $r \in\left[0,1 / 2^{k}\right]$ справедлива оценка

$$
d_{Y}\left(\widetilde{S}_{r}^{\prime \prime}, \text { id }\right) \leqslant d_{Y}\left(\widetilde{S}_{1 / 2^{k}}^{\prime \prime}, \text { id }\right) \leqslant d_{Y}\left(\widetilde{S}_{1 / 2^{k}}^{\prime \prime}, \widetilde{T}_{1 / 2^{k}}\right)+d_{Y}\left(\widetilde{T}_{1 / 2^{k}}, \text { id }\right)<2 \varepsilon .
$$

Пусть $t \in[0,1], t=m / 2^{k}+r$, где $m$ целое, $0 \leqslant r<1 / 2^{k}$. Тогда ввиду инвариантности меры имеем

$$
\begin{aligned}
d_{Y}\left(\widetilde{S}_{t}^{\prime \prime}, \widetilde{T}_{t}\right) & \leqslant d_{Y}\left(\widetilde{S}_{t}^{\prime \prime}, \widetilde{S}_{m / 2^{k}}^{\prime \prime}\right)+d_{Y}\left(\widetilde{S}_{m / 2^{k}}^{\prime \prime}, \widetilde{T}_{m / 2^{k}}\right)+d_{Y}\left(\widetilde{T}_{m / 2^{k}}, \widetilde{T}_{t}\right) \\
& =d_{Y}\left(\widetilde{S}_{r}^{\prime \prime}, \mathrm{id}\right)+d_{Y}\left(\widetilde{S}_{m / 2^{k}}^{\prime \prime}, \widetilde{T}_{m / 2^{k}}\right)+d_{Y}\left(\mathrm{id}, \widetilde{T}_{r}\right)<4 \varepsilon
\end{aligned}
$$

\section{§3. Альтернатива относительно включения в поток}

\section{1. Когомологичность.}

ОПРЕДЕЛЕНИЕ 3.1. Пусть $f, g \in \mathbb{Z}_{2}(X), T \in \operatorname{Aut}(X, \mu)$. Будем говорить, что $f$ когомологично $g$ над $T$ (обозначение: $f \underset{T}{\sim} g$ ), если найдется такая измеримая функция $u(x) \in \mathbb{Z}_{2}(X)$, что для $\mu$-почти всех $x$

$$
f(x)=u(T x)+g(x)-u(x) .
$$

Если $f \underset{T}{\sim} g$, то $T^{f}$ изоморфно $T^{g}$.

Это понятие естественно возникает при рассмотрении корней из косых произведений. Если $Q^{h}=\left(R^{g}\right)^{2}$, то

$$
Q=R^{2}, \quad h(x)=g(R x)+g(x)=g(R x)-g(x),
$$

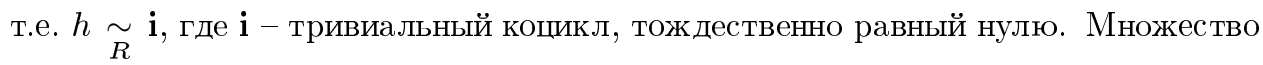
всех коциклов, когомологичных $\mathbf{i}$ над данным $T \in \operatorname{Aut}(X, \mu)$, обозначим через $H_{T}$. 
Лемма 3.1. Множество $H_{T}$ есть всюду плотная подгруппа в $\mathbb{Z}_{2}(X)$.

В [12] отмечено, что если $T$ эргодично, то существуют коциклы, не когомологичные $\mathbf{i}$, причем условие эргодичности можно ослабить. Это утверждение можно уточнить (см. также [13]):

Лемма 3.2. Если $R \in \operatorname{Aut}(X, \mu)$ әргодично, то $H_{R}$ имеет первую категорию в $\mathbb{Z}_{2}(X)$.

Пусть лемма 3.2 доказана и $T \in \operatorname{CAAut}(X, \mu)$. Рассмотрим коцикл $f(x) \in$ $\mathbb{Z}_{2}(X)$, обладающий нечетной аппроксимацией (см. [10]) относительно той же последовательности разбиений, что и $T$, множество таких коциклов обозначим $\mathrm{OAZ}_{2}(X)$. В этом случае $T^{f}$ имеет однократньй спектр, следовательно, $\mathscr{F}\left(T^{f}\right) \subset$ SFlow $(\mathrm{Y}, \nu)$. Пусть $\left\{\widetilde{T}_{t}\right\} \in \mathscr{F}\left(T^{f}\right)$, тогда $f \in H_{T_{1 / 2}}$. Заметим, что преобразования $\left\{T_{t}: t \in \mathbb{R}\right\}$ тоже составляют поток и $T_{1}=T$. Таким образом, справедливо включение

$$
\left\{f(x) \in \mathrm{OAZ}_{2}: \mathscr{F}\left(T^{f}\right) \neq \varnothing\right\} \subset \bigcup_{\left\{T_{t}\right\} \in \mathscr{F}(T)} H_{T_{1 / 2}} .
$$

В силу леммы 3.2 каждое $H_{T_{1 / 2}}$ имеет первую категорию в $\mathbb{Z}_{2}(X)$, а так как по условию доказываемого предложения $\mathscr{F}(T)$ не более чем счетно, то и левая часть (17) есть множество первой категории. Следовательно, таковым является и $\mathscr{K}_{T}$, поскольку отличается от него разве что на множество коциклов, не допускаюших подходящей аппроксимации, а последнее также имеет первую категорию в $\mathbb{Z}_{2}(X)$.

3.2. Доказательство леммы 3.1. Зафиксируем $T \in \operatorname{Aut}(X, \mu)$ и $\varepsilon>0$. Покажем, что для любого наперед заданного $g \in \mathbb{Z}_{2}(X)$ найдется такое $h \in \mathbb{Z}_{2}(X)$, что $d_{c}(g, h \circ T-h)<\varepsilon$. Приблизим $T$ циклической перестановкой $T_{1}$ элементов разбиения $\xi^{l}$ подходящего ранга, a $g-\xi^{l}$-измеримым коциклом $g_{1}$ так, чтобы

$$
\sum_{C \in \xi^{l}} \mu\left(T^{-1} C \triangle T_{1}^{-1} C\right)<\frac{\varepsilon}{2}, \quad d_{c}\left(g, g_{1}\right)<\frac{\varepsilon}{2}
$$

и сумма значений $g_{1}$ по элементам $\xi^{l}$ равнялась нулю $\bmod 2$. Тогда $g_{1} \in H_{T_{1}}$ и найдется такой $\xi^{l}$-измеримый коцикл $h$, что $g_{1}(x)=h\left(T_{1} x\right)-h(x)$. Вычислим, насколько отличаются коциклы $g_{1}$ и $g_{2}=h \circ T-h$ :

$$
d_{c}\left(g_{1}, g_{2}\right)=\mu\left\{x \in X: h\left(T_{1} x\right) \neq h(T x)\right\} \leqslant \sum_{C \in \xi^{l}} \mu\left(T_{1}^{-1} C \Delta T^{-1} C\right)<\frac{\varepsilon}{2} .
$$

Таким образом, $d_{c}\left(g, g_{2}\right)<\varepsilon$, что доказывает лемму.

Отметим, что для суммируемых коциклов со значениями в $\mathbb{R}$ утверждение леммы 3.1 уже неверно.

3.3. Доказательство леммы 3.2. Заметим, что множество $H_{R}$ не может быть массивно. Действительно, в этом случае для любого $g \in \mathbb{Z}_{2}(X)$ массивно множество

$$
g+H_{R}=\left\{g+f, f \in H_{R}\right\},
$$


следовательно, найдутся $f_{1}, f_{2} \in H_{R}$ такие, что $g(x)+f_{1}(x)=f_{2}(x)$. Отсюда $g \in H_{R}$ и, так как коцикл $g$ был выбран произвольно, $H_{R}=\mathbb{Z}_{2}(X)$, что противоречит эргодичности $R$.

Воспользуемся теперь динамической альтернативой, для чего рассмотрим в качестве $\Gamma$ группу $\mathbb{Z}_{2}(X)$, действуюшую на себе следующим образом: элемент $h$ переводит коцикл $f$ в $f+h \circ R-h$. Множество $H_{R}$ инвариантно относительно этого действия и обладает свойством Бэра как образ $\mathbb{Z}_{2}(X)$ при непрерывном отображении $f \mapsto f \circ R-f$. В силу леммы 3.1 орбита коцикла $\mathbf{i}$ при указанном действии плотна в $\mathbb{Z}_{2}(X)$. Используя предложение 1.1 и предыдушее замечание, заключаем, что множество $H_{R}$ имеет первую категорию в $\mathbb{Z}_{2}(X)$.

3.4. Доказательство леммы о сопряженности для $\operatorname{SAut}(Y, \nu)$. Равенство $S^{h}=\widetilde{Q}^{-1} T^{f} \widetilde{Q}$ выполнено в том и только том случае, когда $S=Q^{-1} T Q$ и $h \underset{S}{\sim} f \circ Q$. Пусть $U \times V$ - окрестность в $\operatorname{SAut}(Y, \nu), U \subset \operatorname{Aut}(X, \mu), V \subset \mathbb{Z}_{2}(X)$ - открытые множества. В силу известной леммы Халмоша найдется $Q \in \operatorname{Aut}(X, \mu)$ такое, что $Q^{-1} T Q \in U$. Множество $H_{S}$ плотно в $\mathbb{Z}_{2}(X)$ (лемма 3.1 ), следовательно, найдется коцикл $h \in V$ такой, что $h \underset{S}{\sim} f \circ Q$. Таким образом, $S^{h} \in U \times V$ и лемма 2.1 доказана.

\section{§4. Заключительные замечания}

Пусть натуральное $p \geqslant 2$. Обозначим через $\sqrt[p]{T}$ множество корней степени $p$ из преобразования $T$. Из теоремы 1.3 вытекает следующее утверждение.

Теорема 4.1. Для типичного преобразования $T \in \operatorname{Aut}(X, \mu)$ множество $\sqrt[p]{T}$ имеет мощность континуума.

Рассуждая аналогично доказательству теоремы 1.3 , легко установить, что геометрически различные корни также спектрально неэквивалентны.

Теорему 4.1 можно доказать и непосредственно, применив изложенную схему рассуждений с использованием теоремы 1.4. Рассмотрим косые произведения с коциклами $f: X \rightarrow \mathbb{Z}_{p}$. Если $Q^{h}=\left(R^{g}\right)^{p}$, то

$$
Q=R^{p}, \quad h(x)=\sum_{k=0}^{p-1} g\left(R^{k} x\right) .
$$

В этом случае по-прежнему $h \underset{R}{\sim} \mathbf{i}$, когомологию осушествляет функция $u(x)=$ $-\sum_{k=1}^{p-1} k g\left(R^{k-1} x\right)$.

ПРЕДЛОЖЕНИЕ 4.1. Множество

$$
\mathscr{K}^{\prime}=\left\{T^{f} \in \operatorname{SAut}(Y, \nu): \sqrt[p]{T^{f}} \neq \varnothing\right\}
$$

массивно в $\operatorname{SAut}(Y, \nu)$.

ПреДЛОЖЕНИЕ 4.2. Пусть $T \in \operatorname{CAAut}(X, \mu)$ u $\sqrt[p]{T}$ не более чем счетно. Тогда множество $\mathscr{K}_{T}^{\prime}$ коциклов $f$, для которых $\sqrt[p]{T^{f}} \neq \varnothing$, имеет первую категорию в $\mathbb{Z}_{p}(X)$.

Для доказательства предложения 4.1 достаточно установить, что множество точек локальной плотности отображения $T^{f} \mapsto\left(T^{f}\right)^{p}$ массивно в $\operatorname{Aut}(Y, \nu)$. Для $p=2$ это утверждение содержится в лемме 2.5. 
Доказательство предложения 4.2 аналогично рассуждениям из § 3: вместо (17) нужно рассмотреть включение

$$
\left\{f \in \mathrm{OAZ}_{p}(X): \sqrt[p]{T^{f}} \neq \varnothing\right\} \subset \bigcup_{R \in \sqrt[p]{T}} H_{R}
$$

Результат настояшей работы использован С.В. Тихоновым в [14] для решения поставленной в [11] проблемы о продолжении действия решетки до действия $\mathbb{R}^{d}$. Типичность континуальной периодической части централизатора установлена также О.Н. Агеевым в дополнение к [15] (см. [16]).

Настоящая работа в части теоремы 4.1 выполнена в 2000/2001 академическом году при частичной поддержке РФФИ. Теорема 1.3 и метод ее доказательства анонсированы авторами в [17].

Авторы благодарят Сергея Тихонова за плодотворные обсуждения по теме настоящей публикации.

\section{Список литературы}

1. Халмош П.Р. Лекции по эргодической теории. Ижевск: Регулярная и хаотическая динамика, 2001.

2. Степин А. М. О корнях квадратных из метрических автоморфизмов // Докл. АН СССР. 1967. T. 176. № 5. C. 1023-1026.

3. Chacon $R$. V. Transformations having continuous spectrum // J. Math. Mech. 1966. V. 16. № 5. P. 399-415.

4. Ornstein D.S. On the root problem in ergodic theory // Proceedings of the Sixth Berkeley symposium on mathematical statistics and probability (Univ. California, Berkeley, Calif., 1970/1971). V. 2. Berkeley, CA: Univ. California Press, 1972. P. 347-356.

5. Степин A. M. Применение метода периодических аппроксимаций в спектральной теории динамических систем // Дис. ... канд. физ.-матем. наук. М.: МГУ, 1968.

6. Куратовский K. Топология. Т. 1. М.: Мир, 1966.

7. King J.F. The generic transformation has roots of all orders // Colloq. Math. 2000. V. 84/85. P. 521-547.

8. Glasner E., King J. F. A zero-one law for dynamical properties // Contemp. Math. 1998. V. 215. P. 231-242.

9. Окстоби Дж. Мера и категория. М.: Мир, 1974.

10. Каток А.Б., Степин А.М. Аппроксимации в эргодической теории // УМН. 1967. T. 22. № 5. C. 81-106.

11. de la Rue T., de Sam Lazaro J. Une transformation générique peut être insérée dans un flot // Ann. Inst. H. Poincaré Probab. Statist. 2003. V. 39. P. 121-134.

12. Степин A. M. О когомологиях групп автоморфизмов пространства Лебега // Функц. анализ и его прилож. 1971. Т. 5. № 2. С. 91-92.

13. Lemanczyk $M$. Extensions of cocycles for hyperfinite actions and applications // Monatsh. Math. 1997. V. 123. № 4. P. 209-228.

14. Тихонов $C$. B. Типичное действие групшы $\mathbb{Z}^{d}$ вкладывается в действие группы $\mathbb{R}^{d} / /$ Докл. РАН. 2003. Т. 391. №1. С. 26-28.

15. Агеев O.H. Типичный автоморфизм пространства Лебега сопряжен с $G$-расширением для любой конечной абелевой группы $G$ // Докл. РАН. 2000. Т. 374. № 4. С. 439-442.

16. Агеев О.Н. О типичности некоторых неасимптотических динамических свойств // УМН. 2003. Т. 58. №1. С. 177-178.

17. Степин A.M., Еременко A. М. Типичное сохраняющее меру преобразование имеет обширньй централизатор // Докл. РАН. 2004. Т. 394. №6. С. 739-742. 